\title{
Energy performance assessment of building systems with computer dynamic simulation and monitoring in a laboratory
}

\author{
A. García Tremps \& D. Mora \\ AIDICO, Instituto Tecnológico de la Construcción, \\ Área de Construcción Sostenible, Spain
}

\begin{abstract}
Society is under constant development and it directly entails greater comfort demands for daily activities; these demands are reflected in a continuous increase of energy consumption that is used to satisfy them. According to EU policies to reduce emissions of greenhouse effect gases and considering that the building sector in Spain consumes almost 20\% of total primary energy, new solutions are required to be applied in the existing buildings, given that using passive architectural systems can reduce up to $75 \%$ of energy building demands.

In this context, this project focuses in the study and quantification of the energy performance of the building systems and construction elements. Two methods lead to this quantification: energy dynamic simulation with PC software and energy monitoring in eeLAB laboratory. Comparing real data with simulation data will help to know both possible deviations of in situ test - due, for example, to small deviations in measuring instrumentation, as the limits or fluctuations in the software simulation results.

To obtain real data, a proper enclosure was developed for data gathering (eeLAB) as a test equipment based on an open wireless protocol, which allows it to be easily implemented.

The analysis of data obtained from the two methods will allow us to parameterize energetically the building systems most commonly used in construction. This quantification will provide the different agents of building process with objective criteria for selecting building products and building systems more energetically efficient and appropriate for each location and orientation of the building.
\end{abstract}

Keywords: energy efficiency, building envelope, energy monitoring, simulation. 


\section{Introduction}

Society's environmental concerns and the need for a rational use of energy resources has led European countries to establish strategies compatible with sustainable development in accordance with the Kyoto Protocol to reduce emissions of gases that cause global warming. In fact, the EPBD [1] aims to curb the energy demand of buildings and therefore reduce consumption, as this is directly proportional to economic development and the level of $\mathrm{CO} 2$ emissions.

The construction sector has been one of utmost importance for the Spanish economy, but at the same time has provoked serious impacts on the environment; it is estimated that more than $20 \%$ (EUROSTAT [2]) of the primary energy consumed in Spain is by the construction sector, therefore reductions in demand and energy savings are top priorities to be able to comply with the international energy challenges facing Spain.

This building development has carried with it an increase in the consumption of energy, due largely to the scant attention paid to the incorporation of passive strategies in building, entailing the need to employ active systems in buildings to achieve the level of comfort demanded by today's society. A building designed with energy efficiency criteria can achieve savings of up to $70 \%$ (IDAE [3]) in air conditioning and lighting.

Passive strategies consist in maximizing heat gains and minimizing energy losses of the building in winter, and minimizing heat gains and maximizing the losses of the building in summer. It is important to know the conditions of the environment of the buildings as well as the use they will have to prioritize between the different strategies since some are more recommended for one season than another. In many circumstances incorporating mechanical or electrical devices can improve and enhance some passive strategies, allowing the suitability of different strategies throughout the year.

It is within this context that the idea of the project CESCon has its origins. Its objective is to reduce energy demand in buildings of new construction or rehabilitation by means of the quantification of energy saving due to the use of passive strategies.

Much is said about energy efficiency and demand reduction in construction, but there is a lack of specific studies which recommend or prioritize passive strategies to achieve these goals. The fact that this project not only analyzes passive strategies but also simulates and quantifies them in different scenarios, views compatibility between different strategies, and selects the most efficient choice, entails a degree of innovation and a further step to promote its use and achieve the objective of having an existing building stock of lower consumption thereby reducing greenhouse gas emissions.

Quantification of energy saving measures is made by two independent but related systems, computer simulation and energy monitoring. The latter will be conducted in an experimental laboratory by means of a wireless monitoring system. In order to scale the findings/results of the study on actual buildings, a study is also required on how this same system could be used to measure the compliance of the environmental parameters and constructive systems to the 
Energy Efficiency in Existing Buildings Certification, although in Spain this is not yet regulated by law.

The simulation enables the design of reduced energy consumption and energy efficient buildings and the monitoring measures the quality of the finished project. In addition, the comparison of the simulation model with the real data obtained will show any deviation between both and verify the suitability of the analyzed systems.

The project has been planned so that the results can be used by different agents from the building sector who can count on the knowledge of a proven and reliable technical basis for choosing the most efficient system.

\section{Completion of the project}

The growth of surface constructed buildings in Spain in the period 1990-2005 (prior to approval of the Technical Building Code) was 143\%, which meant a $9.5 \%$ average annual growth experienced in this sector during this period (IDAE [5]). Consequently, the consumption of installations (ACS, heating, cooling and lighting) associated with the building sector has increased, both in the tertiary and domestic sectors; final energy consumption in the building sector is $17 \%$ of the national total (MITyC [4]). This situation is framed within the context of a national dependence on imported energy of more than $80 \%$ (IDAE [5]).

The end of the era of cheap fossil fuels is approaching. During this time, a large quantity of mechanical and electrical equipment to condition buildings has been developed. One of the consequences of placing such importance onto the installations was that passive strategies were relegated to the background, and designers left full responsibility for indoor air quality control to thermal installations.

There is no doubt of the prime need to ensure that rehabilitated or newly constructed buildings consume the least amount of energy possible. This is achieved by initially reducing the energy demand of the building, through responsible design appropriate both to the climate as well as utilization (using passive strategies will result in the greatest savings). Then the installations should be analyzed to ensure that they are the most appropriate and most efficient. Finally, the required energy should come from renewable energy sources which, if feasible, will be integrated into the same building.

This project is within the framework of the reduction of energy demand. Currently, the building sector mentions energy efficiency and demand reduction, but there is a lack of studies that advise or prioritize passive strategies to achieve this goal. The fact that this project not only analyzes the passive systems but also simulates them in different scenarios, finding compatibility between different strategies, entails a degree of innovation and a further step to promote their use and achieve the objective of having an existing building stock of lower consumption thereby reducing the emission of greenhouse gases.

Promoting the reduction of energy demand and quantifying it has been considered of great interest and relevance. Both building design and the incorporation of passive systems can improve energy efficiency in existing 
buildings and new constructions. To offer the same conditions of comfort, a passive measure is more efficient than an active measure because the first affects the second resulting in effective energy savings.

Thus, the need was seen to study the different passive systems available on the market which would be used depending on the type of climate; the aim being to reduce energy demand without renouncing user comfort and well-being.

\subsection{Background}

This is a multi-year project, which focuses attention on aspects related to energy demand during the use of the building.

A significant reduction in energy demand in buildings is achieved through the application of passive measures. This project has a main objective to quantify measures of energy saving applicable to the buildings, through the study and analysis of data obtained through measurements of a real physical model.

The possibility of incorporating the findings of the project to actual buildings would make it possible to obtain energy efficient buildings. The study of the behavior of passive systems in construction is considered important in the building sector, due to the wide variety of constructive solutions with unknown energy behavior. It is therefore necessary to study this behavior and quantify the energy reduction of each system because companies in the construction sector are at present obliged to adapt to the new laws from Europe insisting on energy saving.

The possibilities that this project offers to companies related to the building sector are evident. Thanks to proven results, a reasonable and reasoned election of a passive system or tested strategy can be made, that best suits the building typology, and therefore greater improvements in energy efficiency will be produced throughout the life of the building.

Therefore, the most important specific objectives which the implementation of this project aims to achieve are:

- Use the assessments obtained by monitoring the different constructive systems to provide guidance regarding the most appropriate systems to apply to different scenarios and explore compatibility.

- Compare real data with data obtained through simulation to know both possible deviations from the results of the tests in situ, as well as fluctuations or limits in the results of programs used in computer simulation.

- Prepare a technical report as a guide for the selection of constructive systems intended to satisfy comfort needs.

In addition, given the impact of the energy issue on the environment, it is considered important to underline the specific environmental objectives of the project:

- Reduce greenhouse emissions. Passive techniques to reduce GHG emissions in buildings are studied in this project, so as to encourage the use of these passive strategies for reducing energy consumption.

- Quantify the reduction of energy through passive strategies. Due to the 
great unawareness that currently exists regarding the impact of passive systems on energy expenditure, the implementation of this project is intended to perform a valuation that allows the selection of the best system depending on building typology and location.

- Promote good environmental practices in the construction sector. Much of the energy consumed in buildings is due to a citizen's lack of awareness of energy wastage while achieving the desired comfort conditions. The good use of passive systems by the citizen in their home or workplace would result in a substantial reduction in energy consumption, without prejudicing comfort levels.

The research project CESCon (2009) and the subproject CESCon 2 within project ME3CO (2010) are regional I+D Projects, developed by AIDICO (Construction Technology Institute) and funded by IMPIVA (Institute of Small and Medium Industry of the Valencia Regional Government), through ERDF (European Regional Development Fund).

\section{Conception and composition of the system}

For the implementation of the project a model type (enclosed area) has been selected that allows the study under normal use conditions; different aspects of geometry, constructive systems and materials have been analyzed for this purpose. To allow comparative analysis of different constructive solutions in identical weather conditions, two modules have been used in the project in the same position and orientation. These two modules form the experimental laboratory eeLAB.

\subsection{Model type development}

The eeLAB laboratory consists of two modules for trials; with a north-south orientation. Using simulation programs to optimize the best location search according to the available environment, the positioning of the study models (modules) is set in such a way that they are not affected by exterior shadows, or shadows between them.

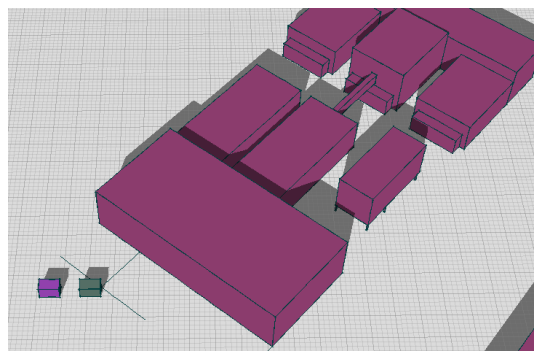

Figure 1: Computer simulation. 
The geometry of the modules is linear, with dimensions of $4.40 \mathrm{~m} \times 2.40 \mathrm{~m}$ floor and $2.30 \mathrm{~m}$ height. Its surface of approximately $10 \mathrm{~m} 2$, allows the volume to resemble a room. Additionally, modular material for compact volume has been used to allow any eventual changes to be quick and economical.

Each side of the module, except for the south side which is the testing side, has $12 \mathrm{~cm}$ insulation to reduce heat flow on these sides, thereby causing the heat flow to occur mainly on the testing face. Although $0.12 \mathrm{~m}$ thick insulation is not excessive for certain latitudes, this laboratory is located in Paterna (Valencia, Spain), with a Mediterranean climate, so this is greater than the insulation that is often used in most construction systems $(0.03$ to $0.06 \mathrm{~m})$ and those abiding by the Technical Building Code for this climatic zone B3 (Valencia, Spain).

The reason the south face is the testing face is because it receives more thermal oscillations during the day, due to the influence of solar radiation on the enclosed area, allowing a multitude of heat transfer analysis. At the same time, the other five faces of the module are also monitored, so as to maintain total control over the flow of heat that exists in all of them.

The proposed solutions have been elaborated in a computerized presimulation to obtain noticeable changes in the various options measured. The resulting model comes from the study of geometry, materials and their orientation and location in the environment.

The project testing module has been composed using this information. Prefabricated material has been chosen for speed and because it allows the change of constructive systems that are going to be analyzed without affecting the rest of the module.

The following elements have been acquired for its composition:

- Sandwich Panel $0.04 \mathrm{~m}$ thick, $2.3 \mathrm{~m}$ height, ribbed outer skin and corrugated inner skin: 22 units

- Sandwich Panel $0.08 \mathrm{~m}$ thick, ribbed outer skin and corrugated inner skin: 16 units

- Outside door 1 blind panel leaf, frame white aluminum and leaf sandwich panel: 2 units

- Insulation covered with injected polyurethane $0.120 \mathrm{~m}$ thick: 2 units

- Phenolic Enamel Board: 2 units

- Floor base without board with polyurethane injected insulation $0.12 \mathrm{~m}$ thick: 2 units

- $\quad$ Sandwich Panel enclosure 0.08m thick: $4.36 \mathrm{~m}$

- Ventilated façade panel made with aluminum structure and fiberglass thermoset resin soffits: 1 unit

- Sliding window two leaves of $1.5 \times 1 \mathrm{~m}$ White aluminum glazed, 4-6-4 transparent without bars: 2 units

\subsection{Monitoring and simulation}

Climatological parameters (atmosphere temperature, relative humidity, speed and direction of the wind and radiation) and thermal parameters (temperature inside enclosed area, surface temperature on each layer of the module, relative 
humidity, and heat flow of the module) are measured in monitoring tests in situ. Additionally they can be calculated by the simulation programs to compare the results.

After an analysis of the measure parameters and the most suitable sensors, the different monitoring methodologies were studied, reviewing the compatibility between the various components of the measure instrumentation. The measure parameters, referring to the energy saving passive measures of the building, were channeled into an open, easily implementable system that would enable the project to rapidly adapt in case of any deviations or future amplifications (i.e. more parameters to monitor or adaptation to an extended environment). Additionally there is the opportunity to expand the system in the long-term with internal environment and internal radiation parameters, along with parameters relating to active systems, parameters of thermal installations, lighting performance and consumption.

The monitoring system has been selected according to the specific needs of the experiment, but always bearing in mind its future applicability to real environment "building" (scalability).

In the first place, the parameters to be monitored in a built environment were selected to evaluate their energy efficiency; they were then transposed to the concrete needs of the system. At the same time, the characteristics of the different monitoring protocols were analyzed so as to compare the detected needs with the characteristics of each one.

A preselection was made of only the most suitable systems to be used in the study environment, or ones that with reasonable environmental modifications, could be considered.

Thus it was possible to assess the compatibility of each system in the physical environment of the actual place where the measurements would be taken, until an open system was found that would go further than meeting the immediate needs of the project, one that would be easily implementable and even scalable according to the needs identified during the research or in future projects.

Finally, a decision was made to resort to the ZigBee Protocol because of the advantages the WSN (wireless sensors networks) offer to adapt to the demands of the experiment. It also allows a reduction in system installation and costs in new scenarios where it is difficult to implant a system with cables.

The WSN were originally developed for monitoring in the military field; currently the development of these networks focuses on domotic, environmental, health, traffic or structural monitoring applications (Perkins et al. [6]).

In particular the ZigBee Protocol has low energy consumption, which allows the useful life of the batteries of data acquisition modules to be optimized. This feature means that access to the study sites is not necessary for long periods of time; human intervention on the monitorized site always interferes with the capture of data by changing the conditions of the environment.

Using the ZigBee protocol allows you to export the results of research from an experimental and local level to actual buildings, with measures in situ; the advantage of a scalable system is that of being able to adapt dimensions according to the repeater nodes needed to expand the system later. The versatility 


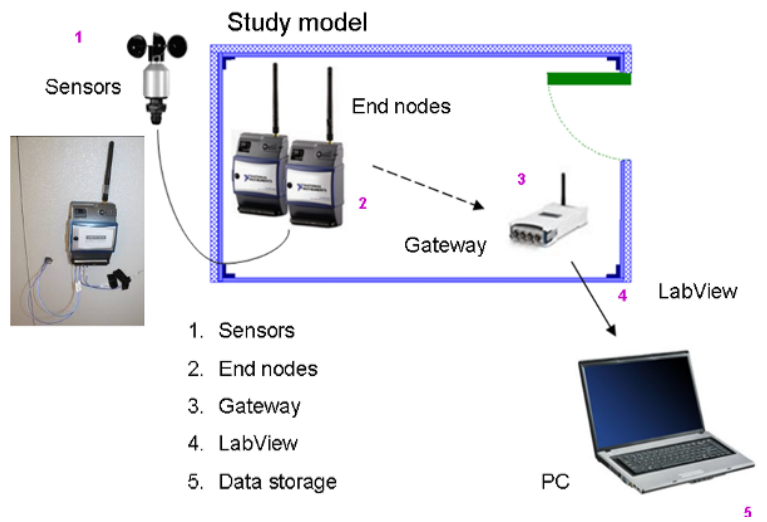

Figure 2: $\quad$ LabView system.

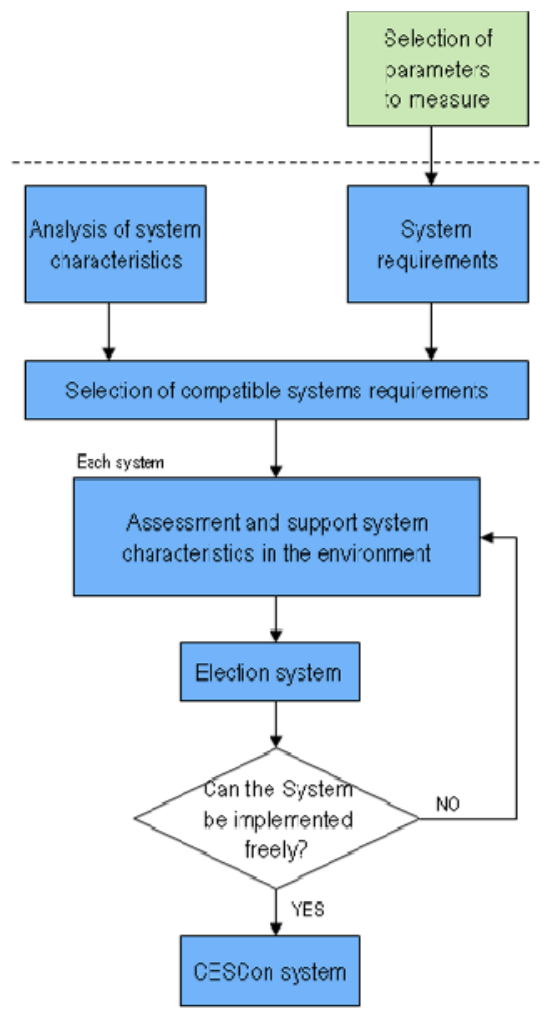

Figure 3: $\quad$ Procedure for election of the measurement system.

of the system supports any analog input and digital output, so it is possible to install dynamic device controls for domotic or active installations. 
This protocol allows an open system in which different types of sensors (thermal radiation, humidity, etc.) can be integrated; these are programmed within the wireless LabView program. The WSN consists of data acquisition modules (end nodes) that are responsible for transmitting the measured values of the sensors to the coordinator (router) that has been programmed with the LabView program for dumping measurements to the database.

Additionally, throughout the project a visualization system Diagmon (www.diagmon.es) has been developed and this is continually expanding its capabilities to include the management and processing of data through a web platform.

The disposition of the sensors is divided mainly into two types; those which collect weather data (temperature, humidity, solar radiation, wind speed and direction) and those that measure the interior parameters of the modules (humidity and temperature within each of them and that of their constructive systems (the heat flow and the surface and interior temperature of each layer of the panels that make up the enclosed area is measured.)

In the search for instrumentation phase, the analysis of compatibility with the physical environment had a favorable comparison, but once the system was started problems occurred concerning the retransmission of the wireless signal to the exterior computer, so the experiment had to be modified and redesigned until reaching the previously commented. The original intention was that the data acquisition modules within the enclosed area would communicate by wireless system with a repeater in the exterior and that would transmit the wireless signal to store data on a computer. Once the system was started, the wireless signal was strongly attenuated by the aluminum shell of the study model. Therefore the issue was resolved by placing the gateway inside the enclosed areas, to communicate wirelessly with the data acquisition modules and by the institute LAN (local area network) with the computer where the measurements are stored. The amendments made to the environment were rapid and did not cause any deviation to the initial timetable.
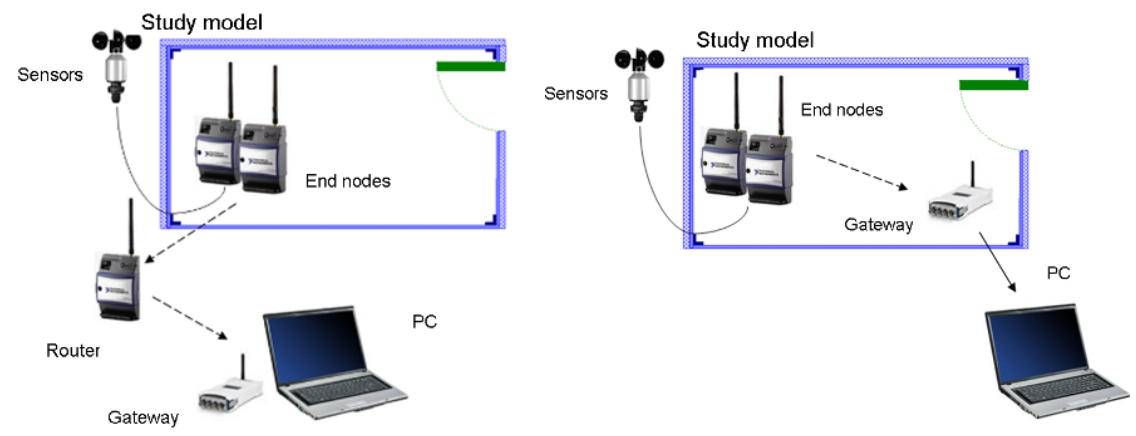

Figure 4: $\quad$ Expected model and realized model. 


\section{Partial results and conclusions}

The analysis process which is being carried out is based both on real measurements as well as computer-assisted simulations. The degree of efficiency of each of the proposed and tested measures may be determined through the analysis of these contrasting results.

From the outset, the thermal bridges in the constructed enclosed areas could be detected by using the thermographic camera. Although the influence was identical in both models, due to the similar disposition, a decision was made to solve those in proximity of the corners, at the joint between panels of different orientation.
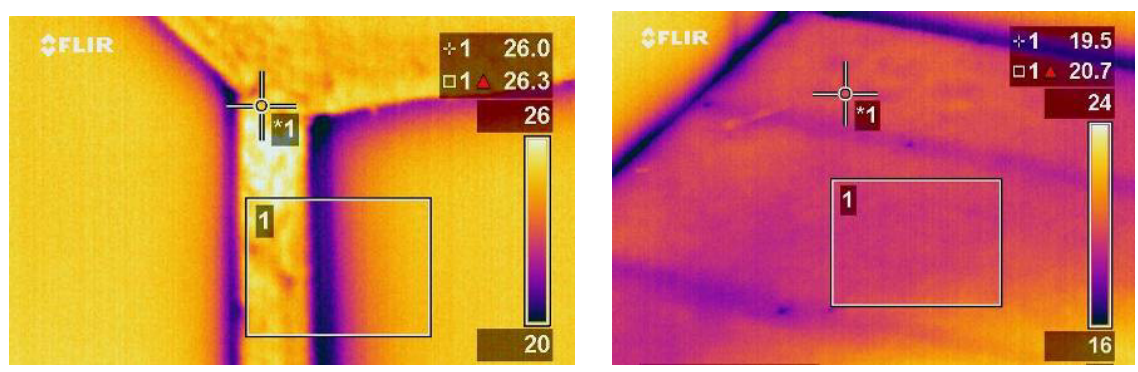

Figure 5: Thermography: corner between vertical walls and horizontal surfaces and soil structure, values in ${ }^{\circ} \mathrm{C}$.

Once the variable of the thermal bridges was homogenized in this way, and after the placement of sensors, we proceeded with the study of the various enclosures. Those tested on the south side so far have been: façade consisting of two sandwich panels, one of $0.08 \mathrm{~m}$ thick and the other of $0.04 \mathrm{~m}$ thick and a façade consisting of only one $0.04 \mathrm{~m}$ thick sandwich panel. The next constructive system to be tested will be a ventilated façade made with aluminum structure and fiberglass thermoset resin soffits.

From a first comparison made between data obtained from monitoring and the results of the simulations, abnormalities have been detected; for example, significant deviations between surface temperatures of the enclosures have been obtained. This is being studied so as to rule out the cause of the deviation being design mistakes of the experiment (this happened at first with the values measured by the thermocouple (temperature) as they were affected by the way it was screwed in).

The dynamic simulation programs that are being used for the simulation of the enclosed area tests are EnergyPlus within the DesignBuilder program.

On the other hand the monitoring system database is being analyzed by PCA, (Principal Component Analysis) which simplifies the structure of the recorded data, and shows the structure of the implicit relationships between variables in order to be able to compare the correlations of each monitoring trial (incident radiation, surface temperature, etc.). 
At the moment the eeLAB is being instrumented with thermal facilities to condition the enclosed area in cooling or heating mode so as to be able to monitor the direct consumption of each passive strategy tested.

In the near future, with the experience gained in this project, an extension to the eeLAB installations is foreseen, which will include simultaneous evaluations of different constructive solutions and consumption monitoring, as well as analysis of the parameters related to gaps and carpentry, not only thermal and energy but also lighting.

\subsection{Project capability}

The eeLAB is an experimental laboratory created to have an enclosed area where actual constructive solutions can be tested and where actual measures from the tests can be compared with simulation data. Its objective is to be able to implement the test conclusions held in the lab, on a larger scale, the "building".

From the comparison between a pair of measurements (those obtained in the enclosed area mentioned and where passive strategy is applied) conclusions may be reached regarding the possibilities that arise in construction to improve energy efficiency in existing buildings. By monitoring a particular constructive system for a limited period of time in the eeLAB, the energy behavior can then be extrapolated throughout the year by computer simulation.

Furthermore, when conducting a dynamic simulation, thermal and energetic behavior of the constructive system is analyzed and this quantifies the different strategies for the analyzed scenarios. At the same time, it will be possible to combine different strategies in the study model, allowing the most efficient choice and leading to a degree of innovation and a further step to promote its use and the objective of having a building stock of lower consumption and therefore a reduction in greenhouse gases.

The project has been designed so that the results can be used by different agents of the building sector: designers who may apply the results to their buildings, developers and building companies, companies engaged in the development of new passive strategies or new technologies who need the knowledge of a proven and reliable technical basis for the choice of the most efficient system. In addition, users of these buildings will achieve a high degree of comfort with a lower consumption of energy which will be reflected in a reduction of energy expenditure.

Companies linked to the construction sector are currently in the spotlight, since they are the ones who must take that big step to investigate and implement real energy saving measures. This project will allow them to be able to reach that target and acquire new skills to improve the ongoing construction-building process, adapting and optimizing passive systems to different typologies and environments of the buildings.

\section{References}

[1] EPBD. Directive 2010/31/EU of 19 May 2010 on the energy performance of buildings. Directive 2002/91/EC of the European Parliament and of the 
Council of 16 December 2002 on the energy performance of buildings. http://ec.europa.eu/energy/efficiency/buildings/buildings_en.htm

[2] Self-processing data of EUROSTAT data. EUROSTAT, Panorama of energy. Energy statistics to support EU policies and Solutions, Office for Official Publications of the European Communities: Luxembourg, pp.48-50, 2009.

[3] Instituto para la Diversificación y Ahorro de la Energía IDAE, Guía práctica de la energía. Consumo eficiente y responsable, p.128, 2010 http://www.idae.es/index.php/mod.documentos/mem.descarga?file=/docume ntos_11406_Guia_Practica_Energia_3ed_A2010_509f8287.pdf,

[4] Ministerio de Indūstria, Turismo y Comercio, La energía en España 2009, División de Información, Documentación y Publicaciones: Madrid, p.195, 2010.

[5] Instituto para la Diversificación y Ahorro de la Energía IDAE, Plan de Acción 2008-2012 de la Estrategia de Ahorro y Eficiencia Energética en España, http://www.idae.es/

[6] Perkins, C., Belding-Royer, E., Das, S. Ad hoc On-Demand Distance Vector (AODV) Routing, 2003. 